\title{
A Numerical Case Study on Contact Analysis with Large Displacement
}

\author{
A. Yamasaki ${ }^{1, a}$, H. Obiya ${ }^{1}$, K. ljima ${ }^{1}$ and Z. M. Nizam ${ }^{2}$ \\ ${ }^{1}$ Department of Civil Engineering and Architecture, Saga University, Honjo, Saga 840-8502, Japan \\ ${ }^{2}$ Faculty of Civil and Environmental Engineering, Universiti Tun Hussein Onn Malaysia, 86400 Parit Raja, Johor, \\ Malaysia
}

\begin{abstract}
In case of geometrically non-linear analysis of plane frame structures, BernoulliEuler beam elements considering the shear deformation to be zero are generally used. But, in node-element contact case, when a contact point is approaching to one of both element ends, diverging of unbalanced forces makes the analysis impossible because of neglecting of the shear deformation. However, in such a case, the element force equation with Timoshenko beam theory which considers the shear deformation is quite effective. In this study, the contact element consistent of both ends and a contact node derived by Timoshenko beam theory is applied to several case of numerical examples. As a result, its convergent performance can be verified in the almost part of element and got accuracy of the solutions.
\end{abstract}

\section{Introduction}

In case of geometrically non-linear analysis of frame structures, the general formulation of the finite element method (FEM) is to derive geometrical stiffness from the relation between strain inside of element's body and displacements of node. On the other hand, the tangent stiffness method (TSM), proposed by Goto, Hane and Tanaka [1], defines elements' behavior in the element force equation. Therefore, the geometrical stiffness, derived by the differential of the equilibrium equation between element edge forces and nodal forces, can express the non-linearity caused by rigid body displacement of each element.

In contact case of node-element or element-element with large displacement, FEM may require so difficult and complex procedures to create a new node when the occurrence of contact. For example, Konyukhov et al. [3] had to use solid elements to simulate a contact model of simple 2-dimensional beam. On the other hand, TSM uses the element force equation, defined in the element coordinate system with a statically determinate and stable support condition. Therefore, it is so easy to add one or more contact points to an element, and to obtain solutions of perfect equilibrium as well as in case of using ordinary element with two nodes of both ends. Nizam et al. [4] applied a contact beam element with three nodes using Timoshenko beam theory to 2-deimensional frame structures. This "Timoshenko contact element" makes it easy to obtain solutions even if when the contact node approaches to an element end, because of effect of shear deformation. In this study, several numerical examples are shown and the rationality of the Timoshenko contact elements is verified.

\footnotetext{
${ }^{a}$ Corresponding author : 15577017@edu.cc.saga-u.ac.jp
} 


\section{Tangent Stiffness Method}

Tangent Stiffness Method (TSM) is a method to evaluate a geometric nonlinearity caused by the rigid body displacement of element, when the element deformation is defined in the element force equation. This makes the unbalanced forces converge by the iteration process that is equal to Newton Raphson method. In addition, strict compatibility and an equilibrium equation are applicable in the iteration process to converge the unbalanced force. Therefore, TSM has an extremely high convergence performance.

\subsection{General formulation}

Here, an element has two ends and the force element vector of both edges are represented by $\mathbf{S}$. Considering a plane coordinate system, if the external force vector is represented by $\mathbf{U}$, and the equilibrium matrix by $\mathbf{J}$, the equilibrium condition can expressed by the following equation:

$$
\mathbf{U}=\mathbf{J S}
$$

By differentiation of Equation (1), the tangent stiffness equation can expressed as:

$$
\boldsymbol{\delta} \mathbf{U}=\mathbf{J} \delta \mathbf{S}+\boldsymbol{\delta} \mathbf{J S}=\left(\boldsymbol{K}_{\boldsymbol{O}}+\boldsymbol{K}_{\boldsymbol{G}}\right) \boldsymbol{\delta} \boldsymbol{u}
$$

Here, the differentiation of Equation (1) simultaneously extracts $\boldsymbol{\delta} \mathbf{S}$ and $\boldsymbol{\delta} \mathbf{J}$ which enables the expression of a linear function of the displacement vector $\boldsymbol{\delta} \mathbf{u}$ in the local coordinate system. Meanwhile, $\boldsymbol{K}_{\boldsymbol{o}}$ represents the element matrix, which also simulates the element behavior corresponding to the element stiffness. Meanwhile, $\boldsymbol{K}_{\boldsymbol{G}}$ is the tangent geometrical stiffness.

\subsection{Equilibrium condition in case of contact problem}

The tangent geometrical stiffness of contact problem can be obtained by differentiating the equilibrium equation. Figure 1 shows the element edge forces for a single contact element. On the other hand, Figure 2 shows the nodal forces. The rotation of point $\mathbf{c}$ is neglected, which the degree of freedom of the node is two.

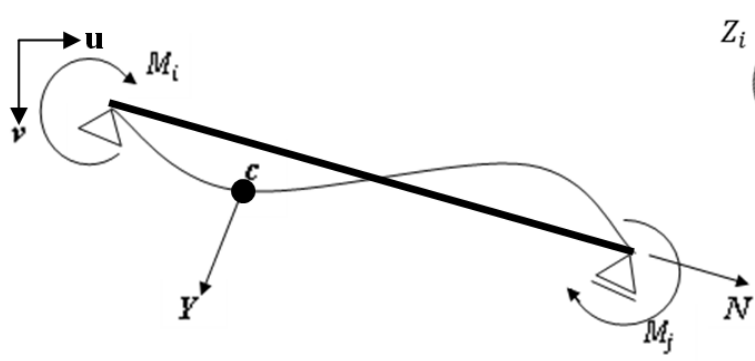

Figure 1. Element edge forces for contact element.

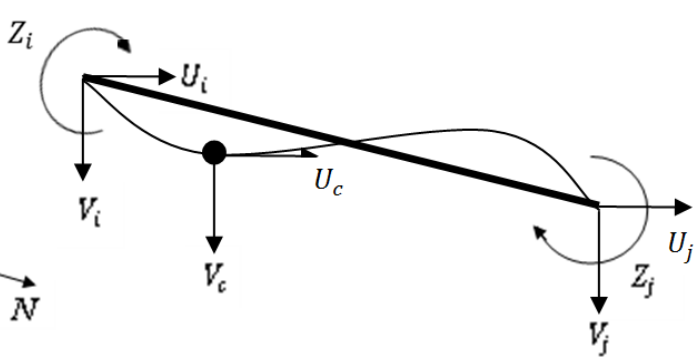

Figure 2. Nodal forces for contact element.

The vector of the element edge forces separate each other and are defined by the following equation:

$$
\mathbf{S}=\left[\begin{array}{llll}
N & M_{i} & M_{j} & Y
\end{array}\right]^{T}
$$


Furthermore, the vector $\mathbf{U}$ can be expressed as following:

$$
\mathbf{U}=\left[\begin{array}{llllllll}
U_{i} & V_{i} & Z_{i} & U_{j} & V_{j} & Z_{j} & U_{c} & V_{c}
\end{array}\right]^{T}
$$

In other words, by differentiating Equations (3) and (4), the tangent geometrical stiffness can be obtained from the equilibrium between $\mathbf{S}$ and $\mathbf{U}$.

\subsection{Timoshenko contact element}

Timoshenko beam elements are effective in case that the shear deformation can not be neglected. Figure 3 shows the equilibrium condition of a beam with elastic and stable support condition under the action of the axial force $N$, edge moments $M_{i}$ and $M_{j}$, and contact force $Y_{c}$. Figure 3 shows the location of the geometric and kinematic variables, and it is assumed that the contact force $Y_{c}$ is within the range of the beam. This local coordinate is a simple but accurate idealization for frictionless nodeelement contact problem. The element force equation of the Timoshenko contact element is given as Equations (5) to (8).

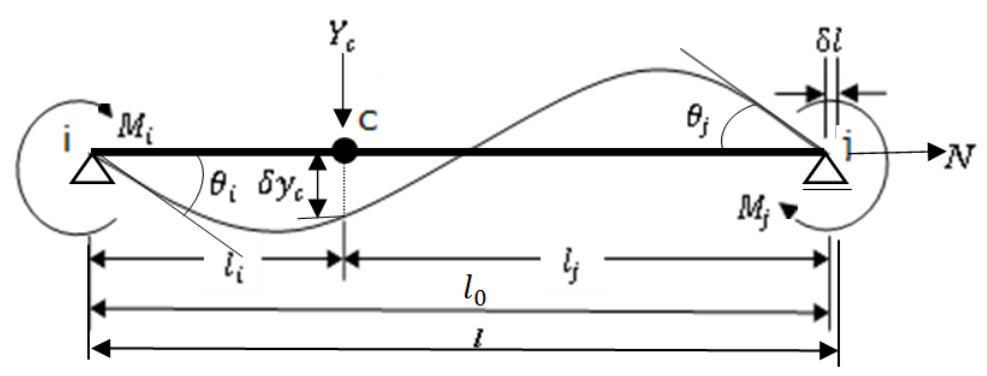

Figure 3. Timoshenko contact element in simply supported beam coordinate.

$$
\begin{aligned}
& {\left[\begin{array}{c}
N \\
M_{i} \\
M_{j} \\
Y_{c}
\end{array}\right]=\left[\begin{array}{cccc}
\frac{E A}{l} & 0 & 0 & 0 \\
0 & \left(4 \Omega+3 l_{i} l_{j}{ }^{3}-108 \Psi^{2}\right) k_{c} & \left\{9\left(l_{0}^{2}-l_{j} \Psi\right)-\Omega\right\} k_{c} & -3 l_{0}\left(l_{j} l_{0}+6 \Psi\right) k_{c} \\
0 & \left\{9\left(l_{0}{ }^{2}-l_{j} \Psi\right)-\Omega\right\} k_{c} & \left(4 \Omega+3 l_{i}^{3} l_{j}-108 \Psi^{2}\right) k_{c} & 3 l_{0}\left(l_{i} l_{0}+6 \Psi\right) k_{c} \\
0 & -3 l_{0}\left(l_{j} l_{0}+6 \Psi\right) k_{c} & 3 l_{0}\left(l_{i} l_{0}+6 \Psi\right) k_{c} & \frac{\left.3 l_{0}+12 l_{0} \Psi\right)}{l_{i} l_{j}} k_{c}
\end{array}\right]\left[\begin{array}{c}
\delta l \\
\theta_{i} \\
\theta_{j} \\
\delta y_{c}
\end{array}\right]} \\
& k_{c}=\frac{E I}{l_{0} \Omega} \\
& \Psi=\frac{E I}{G A_{e}} \\
& \Omega=\left(l_{i} l_{j}\right)^{2}+3 \Psi\left(l_{0}^{2}+l_{i} l_{j}\right)+36 \Psi^{2}
\end{aligned}
$$




\section{Numerical Examples}

\subsection{Contact of a couple of cantilevers}

As shown in Figure 4, there are two cantilevers face each other, which are in contact beforehand. Both cantilevers have 8 equal divisions. Node 1 and node 18 are fixed perfectly, and compulsory displacement is given to $\mathrm{V}$ direction at node 1 . Then, every contact point begins to slide. The material parameters in this example are $E=2.0 \times 10^{7} \mathrm{~N} / \mathrm{m}^{2}, A=3.0 \times 10^{-4} \mathrm{~m}^{2}, I=2.2 \times 10^{-8} \mathrm{~m}^{4}, \mathrm{v}=0.4$ and $\mathrm{G}=7.142 \times 10^{6} \mathrm{~N} / \mathrm{m}^{2}$ respectively. Increment per 1 step of compulsory displacement is $0.208 \mathrm{~m}$.

Figure 5 shows the shape of solution at 10th incremental step, and Figure 6 shows the stable convergent process by TSM. It takes only 9 times of iteration to reduce the unbalanced forces 8 places on its convergent process, in spite of the case that many contact incidents occur simultaneously. Figure 7 shows the solution at 18th incremental step, in which every node approaches to a node on another cantilever extremely. The ratio of $[l j / l]$, which is expresses the position of the contact node on an element (see Figure 3), is 0.9739 . Even in such a complex condition, the solution can be obtained with stable convergent process of only 12 times iterations as shown in Figure 8.

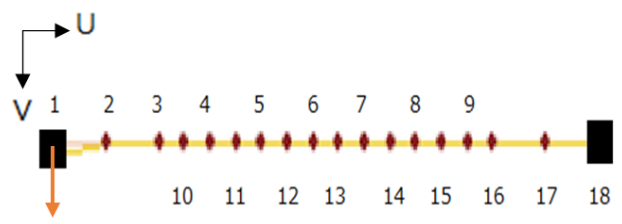

Figure 4. Initial shape.

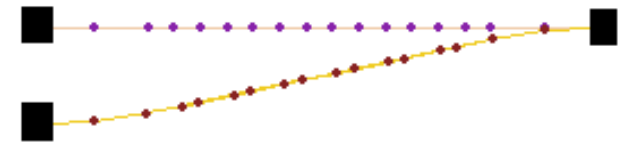

Figure 5. Shape of solution at 10th step.

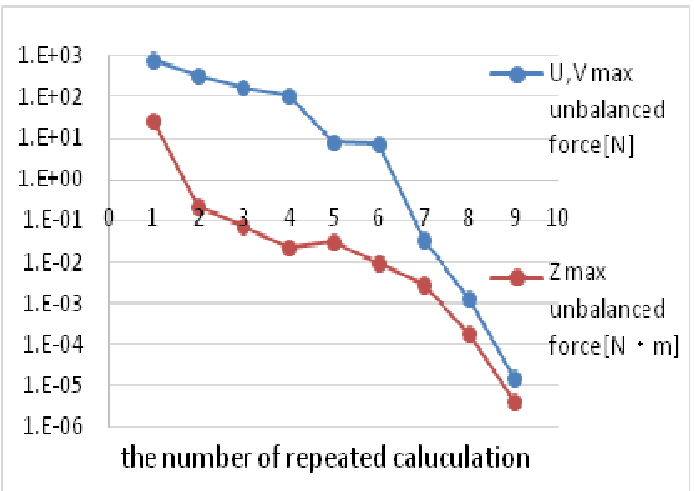

Figure 6. Process of convergence.

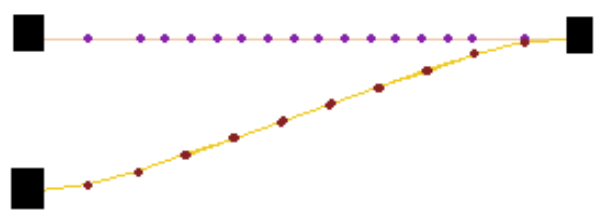

Figure 7. Shape of solution at 18th step.

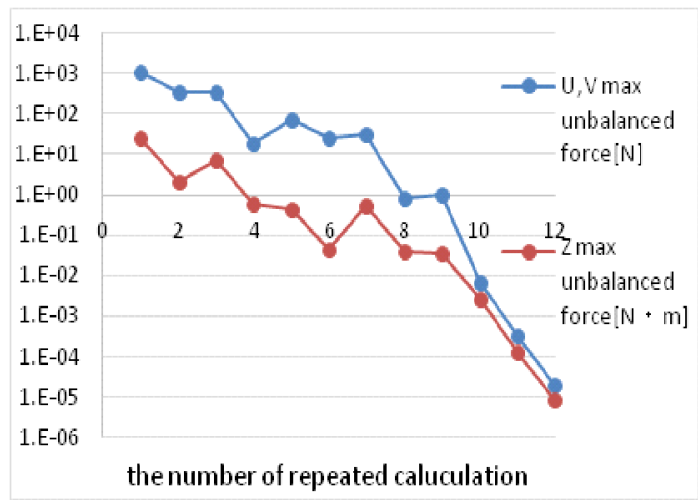

Figure 8. Process of convergence. 


\subsection{Three cantilevers and one contact point}

As shown in Figure 9, this example is consist of three independent cantilevers with 11 nodes and a contact node. Node1, node22, node23 and the contact node are fixed perfectly. The compulsory displacement is given to $\mathrm{V}$ direction at the contact node. The material parameters in this example are $E=2.0 \times 10^{7} \mathrm{~N} / \mathrm{m}^{2}, A=3.0 \times 10^{-4} \mathrm{~m}^{2}, I=2.2 \times 10^{-8} \mathrm{~m}^{4}, \mathrm{v}=0.4$ and $\mathrm{G}=7.142 \times 10^{6} \mathrm{~N} / \mathrm{m}^{2}$. Figure 10 shows the shape of solution when the contact node reached to the cantilever A. Following to it, Figure 11 is the shape of solution when the node 11 on cantilever A contact to the cantilever B, and Figure 12 is when the node 12 on cantilever B contact to cantilever $\mathrm{C}$. When the node12 approaches to the node 30 (Figure 13), the unbalanced force diverged as shown in Figure 14. In such a case, if the incremental calculation is re-started from the previous equilibrium solution, the converged solution can be obtained as shown in Figure 15 by giving larger compulsory displacement and switching the configuration of the contact element to the next door element. This is the simplest procedure to be possible to continue the analysis.
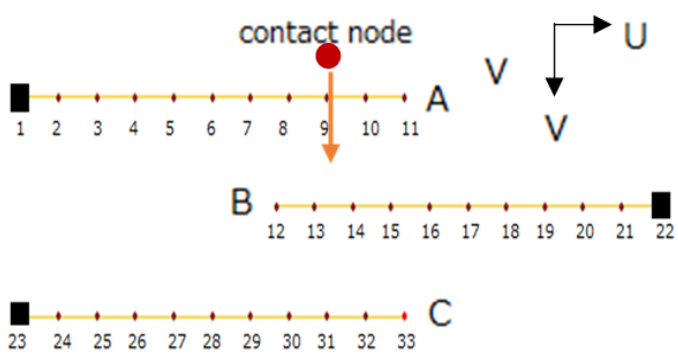

Figure 9. Initial shape.
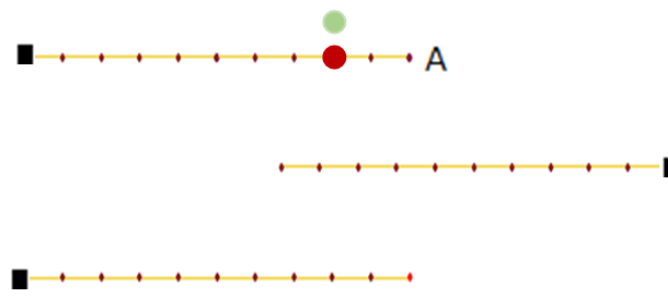

Figure 10. First contact to cantilever A.

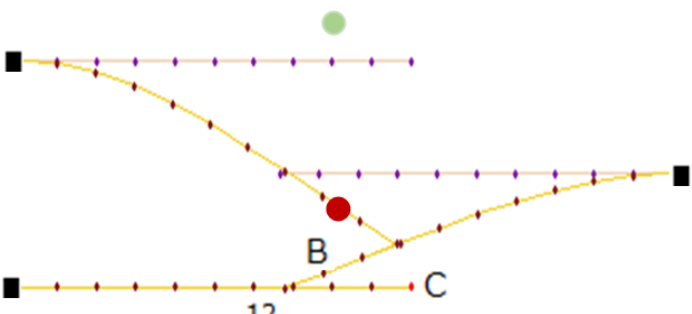

Figure 12. Third contact of B to $C$.

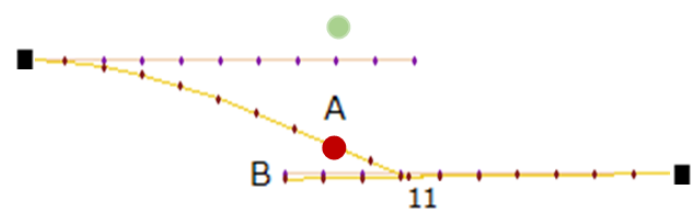

Figure 11. Second contact of A to B.

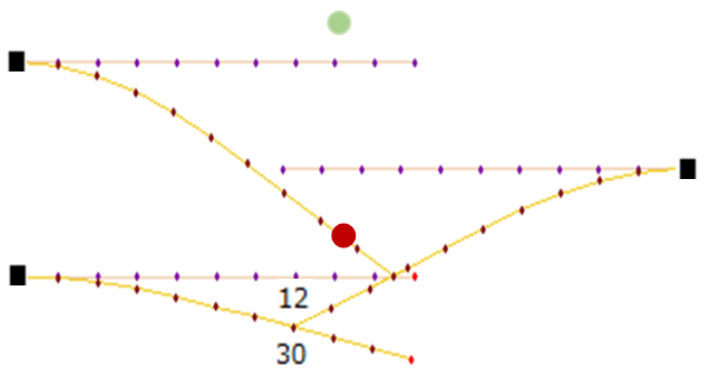

Figure 13. Node12 approaching to Node30. 


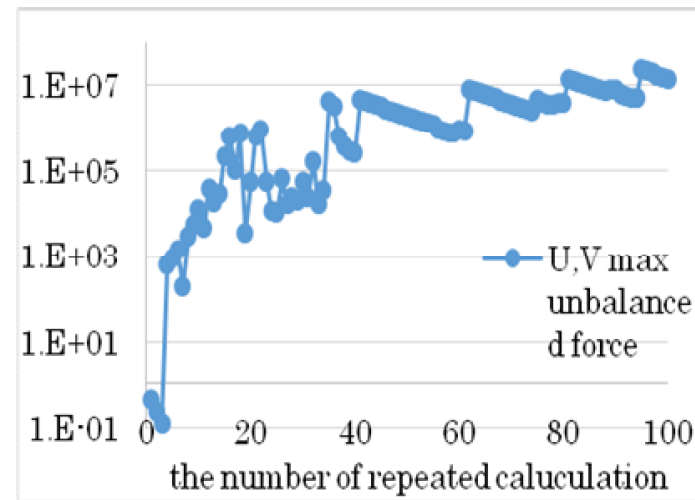

Figure 14. Process of divergence.

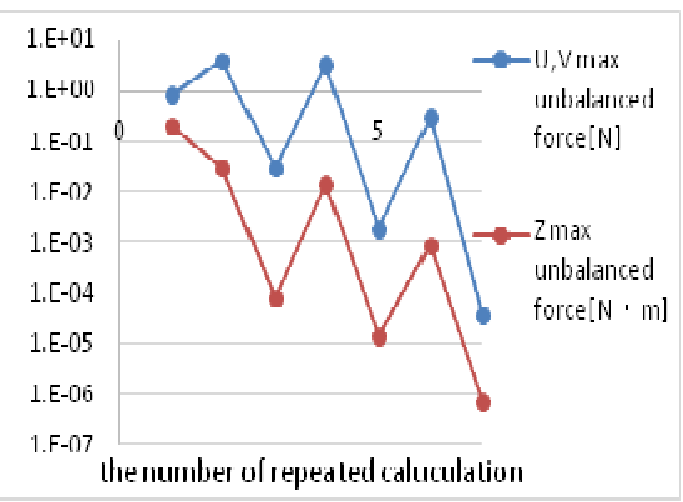

Figure 15. Process of convergence.

The contact node approaches to the end of the cantilever A as shown in Figure16, and then jumps from cantilever A to cantilever B as shown in Figure 17. At the same time, the contact between the contact node and the cantilever A was canceled. On the same process as above, the contact node approaches to the end of the cantilever B as shown in Figure18, and then jumps from cantilever B to cantilever $\mathrm{C}$ as shown in Figure 19. Also in this case, the contact between the contact node and the cantilever B was canceled at the same time. Figure 20 is the solution that the contact node approaches to the end of beam $\mathrm{C}$, and all the contact is canceled in Figure 21.

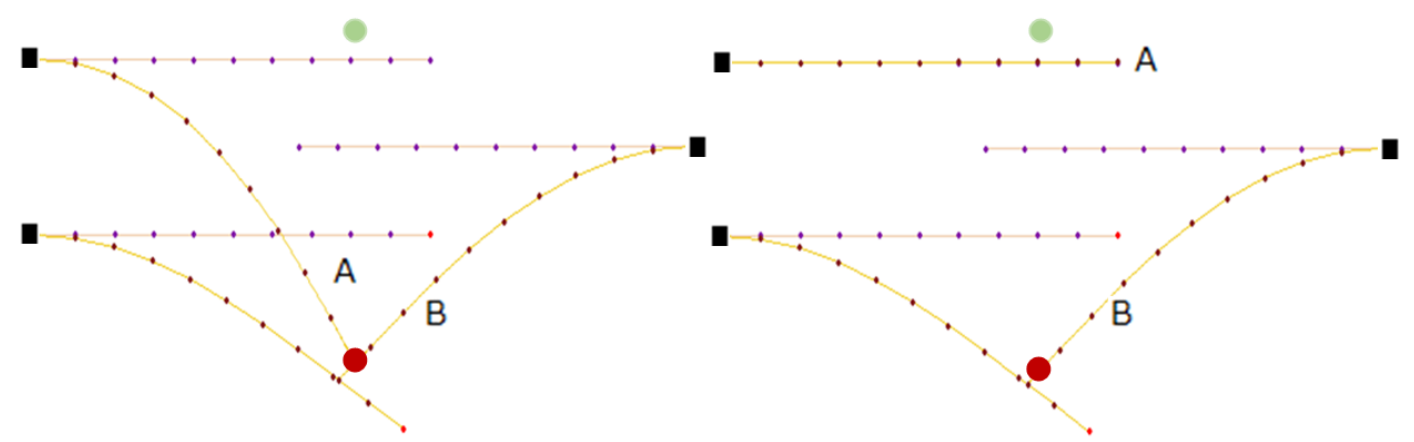

Figure 16. Contact node approaching to end of cantilever A.

Figure 17. Contact node jumping from A to B.
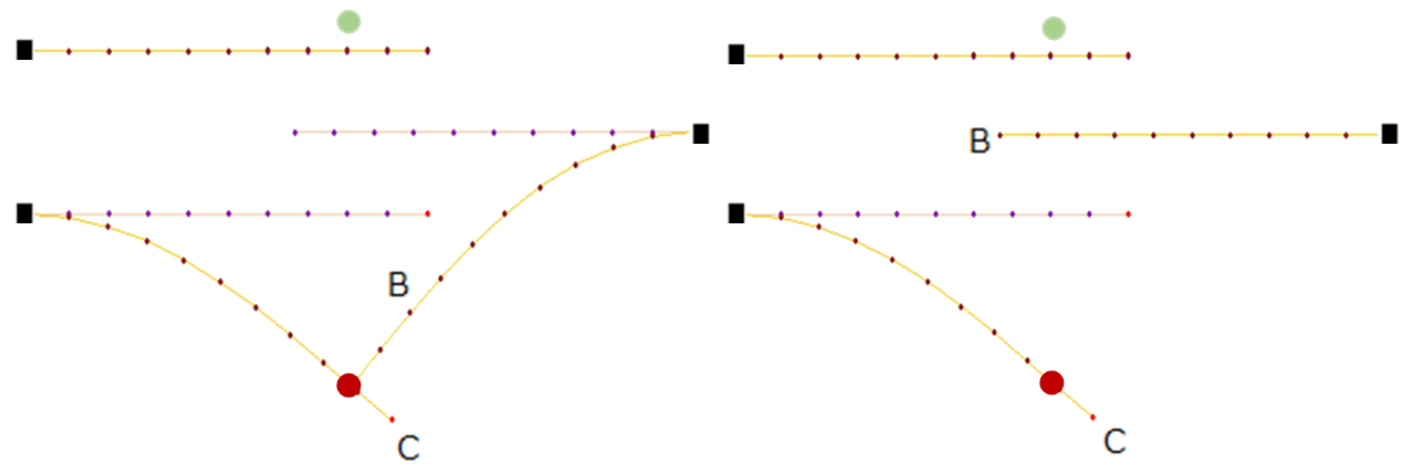

Figure 18. Contact node approaching to end of cantilever B.

Figure 19. Contact node jumping from $B$ to $C$. 


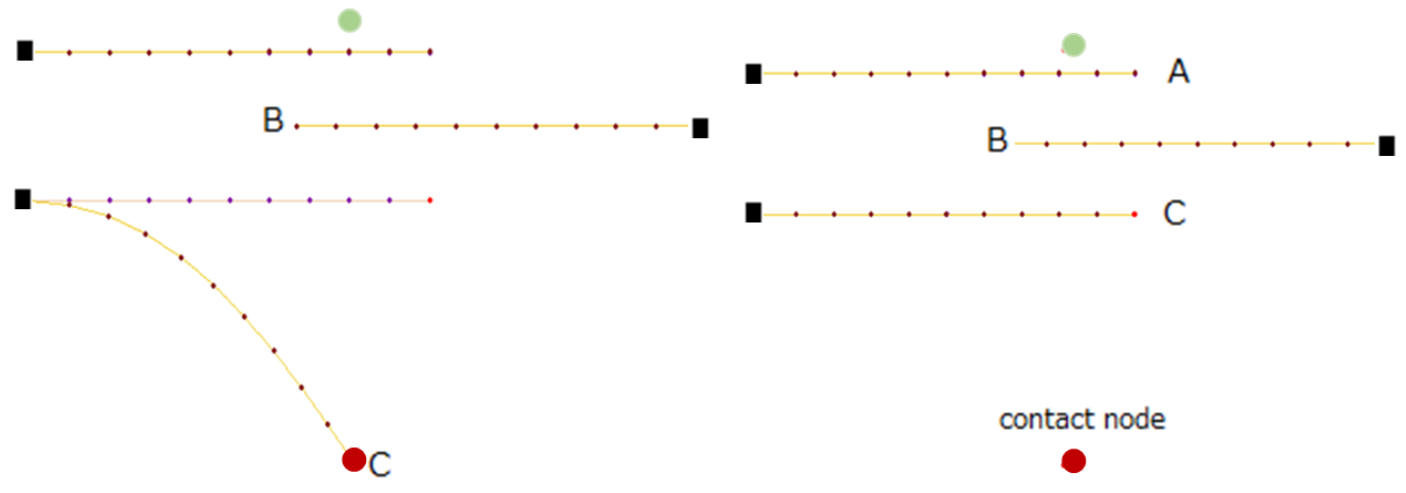

Figure 20. Contact node approaching to end of cantilever B.

Figure 21. Final solution (non-stressed state).

\section{Conclusions}

In this study, two numerical examples are shown and the rationality of the Timoshenko contact elements is verified. In the first example, Even if when the contact node approaches to the end of element and the shear deformation can not be neglected, the converged solution can be obtained by using Timoshenko contact element considered the shear deformation. In the second example, when the contact node approaches to an element end, the unbalanced force diverged. In such a case, the converged solution can be obtained by giving the contact node larger compulsory displacement at the previous equilibrium solution and making it move to the next door element. In another words, distortion problem becomes possible to prevent by using the numerical technique above. As a result, the convergent performance of Timoshenko contact element can be verified in the almost part of element. For the future prospects, it is considered that the converged solution can be obtained on contact analysis with large displacement in 3-dimensional frame structures.

\section{References}

[1] S. Goto, G. Hane and T. Tanaka, The large deformation analysis of plane frame structures by the tangent stiffness method, J. of Japan Society of Civil Engineer, 238, 31-42, (1975).

[2] S. Goto, The pluses and minuses of finite element method in a geometrically non-linear analysis method of structures, <http://www.forum8.co.jp/forum8/ronbun1.htm 1998>.

[3] A. Konyukhov, and K. Schweizerhof, Geometrically exact covariant approach for contact between curves, Computer Methods in Applied Mechanics and Engineering, 199(36-40), 25102531, (2010).

[4] Z.M. Nizam, Effect of shear deformation on convergability of simple contact analysis with large deformation, Proc. Asia Pacific Congress on Computational Mechanics and $4^{\text {th }}$ Int. Symposium on Computational Mechanics, Singapore, (2013). 